\title{
Contact Forces Analysis of an Analogous Huygens Pendulum Using Inverted Tooth Chain
}

\author{
Karim H. Shalaby, Simona Lache, and Florin Corciova
}

\begin{abstract}
The paper analyses and brings to light the importance of contact forces of the Inverted Tooth Chain (I.T.C.) plates on the sprocket wheel at different locations on the Analogue Huygens Pendulum, knowing that the contacts between plates and sprocket always cause vibrations and noise. The MSC ADAMS SOLVER/VIEW software, which is based on multibody dynamics, is used for simulating the contact forces. The simulation results lead to the conclusion that the contact between the plates of the inverted tooth chain (I.T.C.) and the sprocket produces elastic-plastic impacts.
\end{abstract}

Index Terms - Rigid multibody dynamics, contact forces, inverted tooth chain (I.T.C.), analogue huygens pendulum.

\section{INTRODUCTION}

Some common problems in chain drive systems, not related to the chain architecture (bush, roller or IT chain) appear due to the contact between the chain elements and the sprocket teeth. Therefore, high contact forces are created that produce vibrations and noise.

Previous attempts to understand the magnitude of these forces were done by R.V. Mulik [1] and Ishimao Masao and Watanabe Hiroyuki [2] for different chain drive systems in the automotive industry. R.V. Mulik's main objectives were to find the tension and contact forces between the chain and the components of the chain system. Ishimao Masao and Watanabe Hiroyuki developed an approach to suppress the noises and vibrations caused by the contacts between the I.T.C. plates and the sprockets and analyzed the tension of the chain as a whole by using a multibody dynamic simulation software LMS motion. They concluded that most of the noises and vibrations were produced by the engagement and disengagement of the plates with the sprockets. The noises and vibrations would increase with an increase in tension on the chain.

In order to better understand the contacts between the plates of the inverted tooth chain (I.T.C.) and the sprocket, a multibody dynamics simulation software MSC ADAMS will be used, assuming that all the bodies (chain elements and sprockets) are rigid. The general purpose is to understand the behaviour in different situations of an I.T.chain by applying different conditions. This study is based on the Analogue Huygens Pendulum effect. The Anologue Huygens Pendulum effect is caused due to a fixed centroid around which the chain oscilates against the sprocket. A

Manuscript received April 19, 2015; revised July 24, 2015.

Karim H. Shalaby is with Transylvania University of Brasov, Romania (e-mail: karim.shalaby@unitbv.ro).

Simona Lache is with the UTB, Romania (e-mail: slache@unitbv.ro).

Florin Corciova was with the Transylvania University of Brasov, Romania (e-mail: corcifor@schaeffler.com). mono-involute motion is being created in Fig. 1.
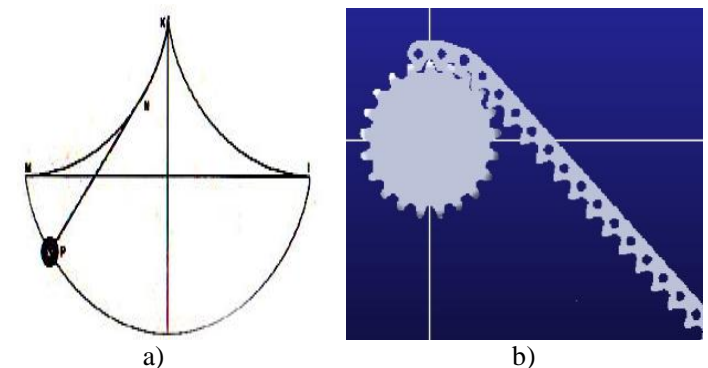

b)

Fig. 1. Huygens Pendulum [3] a); the model of the studied analogue Huygens Pendulum b).

\section{THEORETICAL APPROACH}

Impacts are short lived phenomena of energy, exchanged between colliding bodies. The velocities of colliding bodies change rapidly and the reactions are impulsive in nature. The three types of rigid bodies impacts which have been defined are elastic impacts, plastic impacts and elastic-plastic impacts. In rigid body dynamic models, there are two sets of coordinates which are used when analyzing the displacement of rotation of a rigid body:

1) The global coordinates $(X, Y, Z)$ which are fixed to the ground.

2) The local coordinates $\left(X^{\prime}, Y^{\prime}, Z^{\prime}\right)$ also known as the rigid body coordinate system whose origin is located at its centre of mass.

The equation of unconstrained motion for the rigid body can be written as a set of ordinary differential equations in the following matrix form [4]:

$$
M \ddot{q}=Q_{v}+Q_{e}
$$

where $q=\left[R^{T}, \beta^{T}\right]^{T}$ is the vector of generalized coordinates, $R$ $=\left\{x_{c}, y_{c}, z_{c}\right\}^{T}$ is the vector of the coordinate of the mass center and $\beta=\left\{\beta_{0}, \beta_{1}, \beta_{2}, \beta_{3}\right\}^{T}$ is the vector of Euler parameters. The inertia matrix $\mathrm{M}$ is given by the equation [4]:

$$
M=\int_{\Omega} \rho\left[\begin{array}{lll} 
& I & \\
-G^{\prime} T & \tilde{u}^{\prime T} & A^{T}
\end{array}\right]\left[\begin{array}{lll}
I & -A \tilde{u}^{\prime} & G^{\prime}
\end{array}\right] d \Omega
$$

where the integration is over the entire rigid body, $\rho$ is the density, $I$ is the $3 \times 3$ identity matrix, $A$ is the $4 \times 4$ transformation matrix and is the skew symmetric $\Omega$ matrix obtained from the local coordinates vector u'. The matrix $G$ ' is a $3 \times 4$ matrix expressed in terms of the Euler parameters [5], [6]:

$$
G^{\prime}=2\left[\begin{array}{cccc}
-\beta_{1} & \beta_{0} & \beta_{3} & -\beta_{2} \\
-\beta_{2} & -\beta_{3} & \beta_{0} & \beta_{1} \\
-\beta_{3} & \beta_{2} & -\beta_{1} & \beta_{0}
\end{array}\right]
$$


$Q_{v}$ the first term on the right-hand-side of Eq. (1) represents the vector that absorbs quadratic velocity terms [4]:

$$
Q_{v}=-\int_{\Omega} \rho\left[\begin{array}{lcl} 
& I & \\
-G^{\prime} T & \tilde{u}^{\prime T} & A^{T}
\end{array}\right] \alpha_{v} d \Omega
$$

where $\alpha_{v}=A \widetilde{\omega}^{\prime} \widetilde{\omega}^{\prime} u^{\prime}-A u^{\prime} \dot{G}^{\prime} \beta$ and $\widetilde{\omega}^{\prime}$ is the skew symmetric matrix corresponding to the angular velocity vector $\omega$ ' of the rigid body in the local coordinate system. The "•" denotes the derivative with respect to time. The second term on the right-hand-side of Eq. (1) is the vector of generalized forces [4]:

$$
Q_{e}=\left[\begin{array}{l}
\left(Q_{e}\right) R \\
\left(Q_{e}\right) \beta
\end{array}\right]=\left[\begin{array}{c}
F_{g}+F_{c} \\
G^{T} M_{c}
\end{array}\right]
$$

where $G=A G$ '. The general force includes the gravitational force $F_{g}$ and the impact contact force $F_{c}$ and $M_{c}$ is the vector of the moment of contact force with respect to the mass center. The matrix $M$ is assumed to be positive definite, and Eq. (1) can be written as [4]:

$$
\ddot{q}=M^{-1}\left[Q_{v}+Q_{e}\right]
$$

Introducing the state vector [4]:

$$
U=\left[\begin{array}{l}
\dot{q} \\
q
\end{array}\right]
$$

and the load vector [4]:

$$
R=\left[\begin{array}{c}
M^{-1}\left[Q_{v}+Q_{e}\right] \\
\dot{q}
\end{array}\right]
$$

during impact the contact forces are accompanied by frictions during sliding. These frictions between solids in dry conditions are defined by the laws of Coulomb and give the possibility of studying the elastic and plastic deformations on the surfaces of the two solids in contact, as well as other tribological aspects.

The impact contact force is calculated as the integration of a distributed load over the contact area $\mathrm{S}$ in the following form [4]:

$$
F_{c}=\int_{S} f_{c} d S
$$

where $f_{c}=f_{n} n+f_{t} t$ is the vector of the distributed load. $n$ and ${ }_{t}$ represent the unit vector in the normal and tangential directions, respectively. The normal distributed contact load $f_{n}$ is determined explicitly by the local indentation $\delta$ and its rate of change [4]:

$$
f_{n=\left(k_{G}+c_{G} \dot{\delta}\right) \delta}
$$

The local tangential contact load $f_{s}$ also known as the static friction force is computed using Coulomb's law. When sticking occurs, one has [4]:

$$
f_{s} \leq \mu_{s} f_{n}
$$

where $\mu_{s}$ is the coefficient of static friction. The static friction force $\left(f_{s}\right)$ states the maximum force or the critical force that would prevent the body from moving meaning that the body would start to slide or move when it has overcome the static friction forces. The kinetic friction force $\left(f_{k}\right)$ results when sliding occurs. This is the force reacting inversely to the direction of motion of the solid in contact to the other solid thus overcomming the static friction force [4]:

$$
F_{k}=\mu_{k} f_{n}
$$

where $\mu_{k}$ is the coefficient of sliding friction.

The moment of impact contact force with respect to the mass center is computed as [5]:

$$
M_{c}=\int_{S} m_{c} d S
$$

where $m_{c}=\widetilde{U} f_{c}$, and $\widetilde{U}$ is the skew symmetric matrix corresponding to the local position vector expressed in the global coordinate system. Yet the system consists of constrained contacts as there are revolute joints that constraint the pendulum. Using Lagrange D' Alembert principle we can obtain the following relation [5]:

$$
\{M \ddot{q}-Q\}^{T} \delta q=0
$$

where $Q$ is the vector of all the forces acting on the system excluding the constraint forces as the virtual work principle can be used. The generalized force vector $Q$ includes external applied forces, gravity forces, spring, dampers and friction forces. The constraint equations that describe mechanical joints and specified motion trajectories as well as the contact constraints are expressed in the following equation [5]:

$$
C(q, s, t)=0
$$

where $s$ is the vector of the parameters that describe the geometry of the surfaces in contact.

The virtual changes in the system coordinates and the surface parameters, which are consistent with the kinematic constraints, lead to [5]:

$$
C_{q} \delta_{q}+C_{s} \delta_{s}=0
$$

The final form of a generalized equation of motion of constraint contact forces is described in the following equation [5]:

$$
\delta q^{t}\left\{M \ddot{q}+C_{q}^{T} \lambda-\mathrm{Q}\right\}+\delta s^{T} C_{s} \lambda=0,
$$

where $\lambda$ is a nonzero arbitrary vector.

\section{Modelling AND Simulation}

The contact statements in MSC ADAMS/SOLVER software give the possibility to define two or three dimensional contacts between a pair of geometric objects. The software models the contact as a unilateral constraint meaning that a force has zero values when no penetration exists between the specified geometries, while the force when subjected to penetration between the two specified geometries obtains a positive value. The contact statements in 
the software support the multiple contacts, the dynamic frictions and the contacts between three-dimensional solid geometries [7].

The general settings of calculating contacts in MSC ADAMS/SOLVER software are [8]:

1) The contact kinematics, in which one can define the contact between two geometric objects that are specified in graphic entities.

2) The outward normal definition, which is the calculation of the outward normal of a geometric graphic by defining the type of material and therefore determining the direction of the contact normal forces. For three dimensional solids, which is the case in the present model, the geometric graphics are closed by definition. The outward normal is included in the geometric description and there is no open interpretation in its definition.

3) The contact kinetics where the software classifies contacts into two major types:

- Intermittent contacts which are characterized by the contacts for short intervals of time and are also know as impulsive contacts. The energy loss during the collision is usually modeled as a damping force that is specified with a damping coefficient or a coefficient of restitution. This is the general case of the model presented.

- Persistent contact characterized by the contacts of two bodies for long periods of time. The external forces acting between the two bodies serve to maintain continuous contact.

4) The contact normal force calculation. The software presents two models for calculating the normal force:

- Impact function used in the applied model for simplification in calculations.

- Coefficient of restitution or the POISSON model.

Both models result from a penalty regularization of the normal contact constraints. The penalty of regularization is a mathematical constraint obtained by creating or applying forces along the gradient of constraint. The force magnitude is a function of the constraint violation.

5) The contact friction force calculation. The software uses a relatively simple velocity based friction model for contacts with Coulomb's laws.

\section{RESULTS AND DISCUSSIONS}

The main importance of this study is to show how the contact between the I.T. chain plates and sprocket vary according to the different distances between the plates and the sprocket. Therefore, there is the question of whether all the contacts between the plates and the sprocket will have the same characteristic behavior in different time intervals and with different force values. To illustrate that the system is totally aligned, meaning that the plates are aligned with the sprocket, the top view alignment of the chain plates with the sprocket is taken in consideration, as shown in Fig. 2.

By considering the first set of pairs of I. T. chain plates totally constrained to the sprocket, the possibility of creating contact forces or friction forces between them is eliminated. As the next set of pairs of I.T. chain plates is not constrained to the sprocket, but rather constrained with the rest of the links of the chain, one will notice the contacts between the links and the sprocket as shown in Fig. 3.

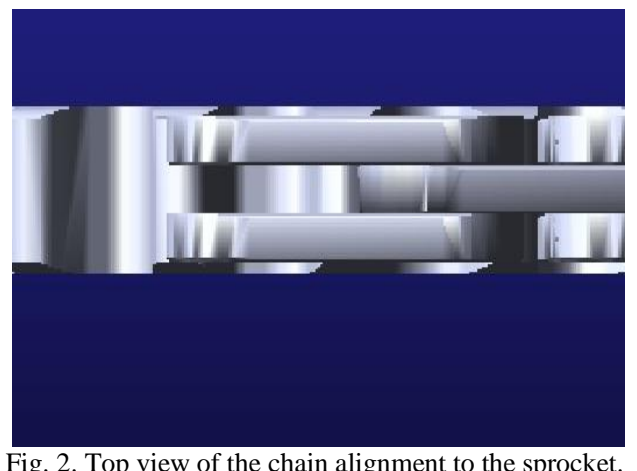

Fig. 2. Top view of the chain alignment to the sprocket.

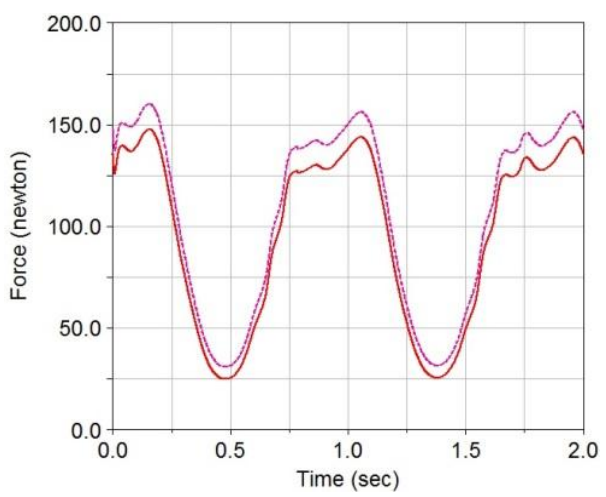

Fig. 3. Contact forces of the second set of pairs.

The distance between the plates and the sprocket is too small obliging the plates and the sprocket to be always in contact. The distance between the plates and the sprocket is too small obliging the plates and the sprocket to be always in contact. This creates friction forces which are permanent and relatively high during the period of oscillation. In general, when the distance between the plates and the sprocket increases, the contact forces of the plates against the sprocket increase whereas the friction forces decrease.

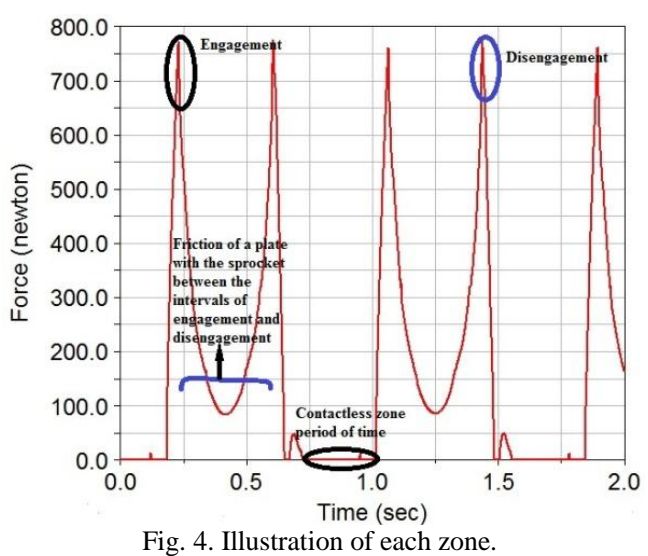

In order to understand the behaviour of the distribution of forces during contact, one can distinctly divide the contacts of a plate with the sprocket into four main zones in Fig. 4:

- the engagement zone where the plate has a first main contact with the sprocket,

- the second zone which clearly shows the friction of a plate with the sprocket,

- the disengagement zone where the plate spontaneously disengages the sprocket and

- the contactless forces zone which marks the end of one oscillating cycle of the plate toward the sprocket. 


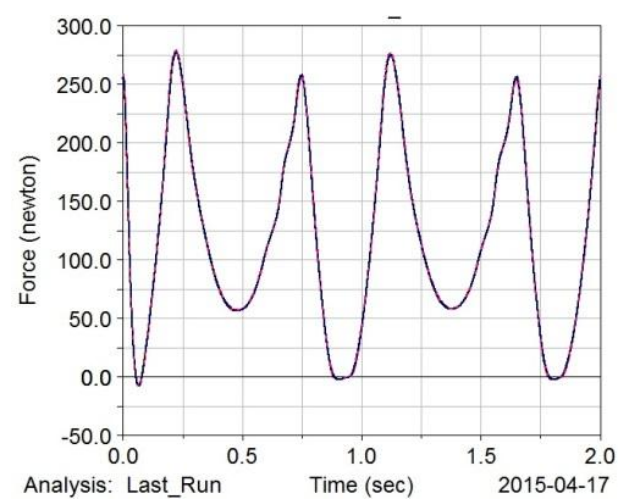

Fig. 5. Contact forces of the third set of pairs.

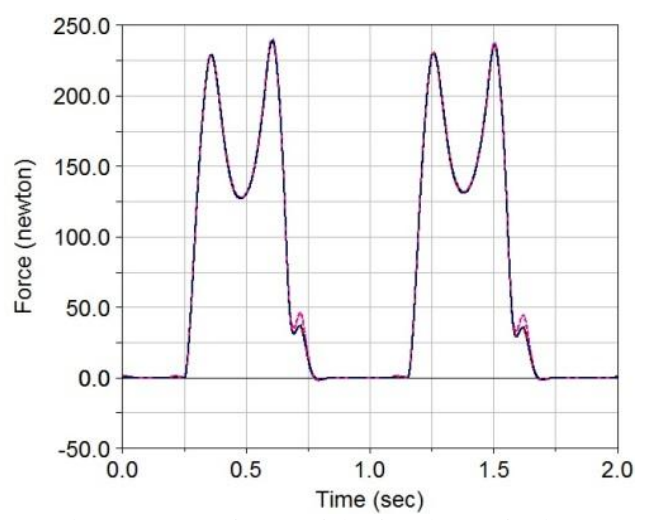

Fig. 6. Contact forces of the fourth set of pairs.

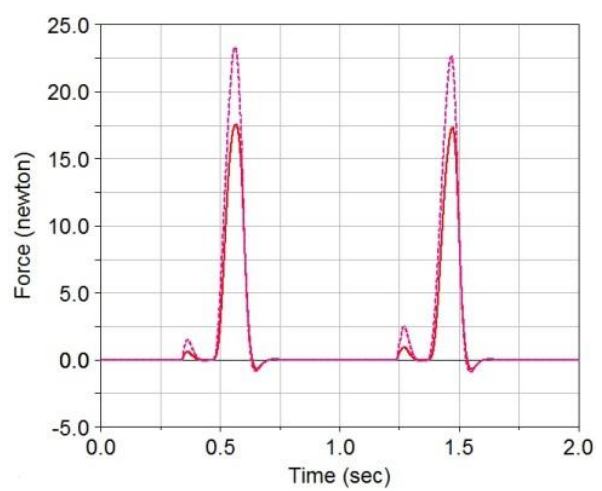

Fig. 7. Contact forces of the fifth set of pairs.

By studying the contact forces along the next links on the chain where the distance of the plates of the links grows further apart from the sprocket, it can be noticed that each set of links has slightly different behaviours. It would have been expected during contact that the engagement force would always be greater than the disengagement force. However, with the decrease of the engagement period of time and the increase of the disengagement period of time while the total contact period of time decreases, the disengagement force tends to be greater than the engagement force represented in Fig. 6. Throughout large contact periods of time the engagement forces between the plates and the sprocket are higher than the disengagement forces, as illustrated in Fig. 5. The friction forces which link the engagement to the disengagement appear in both cases, during large periods of time as in Fig. 5 and small intervals of time as in Fig. 6. Due to the materials damping coefficient the disengagement forces in general should be less in magnitude than the engagement forces, but the disengagement forces are more indirectly dependent on the time period of the contact between the plates and the sprocket. As the total period of time during contact is significantly decreasing impulsive impacts appear in Fig. 7.

\section{CONCLUSIONS}

The contact forces of the chain plates on the sprocket in the Analogue Huygens Pendulum are clearly shown within the simulation presented in this paper. The chain in the Analogue Huygens Pendulum behaves nearly like the system of an automobile, from the cyclic point of view. In the present system the plate takes one cycle to oscillate along the sprocket, whereas in the automobile a plate has to complete a revolution along two or more sprockets. In the Analogue Huygens Pendulum system the contact forces can be divided into four categories:

1) The impulse impacts are shortly lived and the friction forces mainly prevail due to an insignificant distance between the plates and the sprocket.

2) The first intermediate distance shows that the impulses of engagement are higher than the forces of disengagement. Yet, the overall forces would slightly dissipate in time due to the damping coefficient of the materials.

3) The second intermediate distance, slightly bigger than the first intermediate distance, illustrates that the forces of engagement are smaller than the forces of disengagement as the overall period of impact decreases.

4) The distance becomes very large and the impact forces start to fade. The impact is very shortly lived. One can anticipate a reduction in friction forces, therefore the disengagement forces nearly disappear whereas the shocks of previous links of the chain are nearly insignificant, yet still exist.

When the angular displacement increases, the time interval of contact decreases until the pendulum reaches the rest position due to loss of kinetic energy.

It is important to show how the contact forces between a plate and the sprocket actually behave in different I.T.C. systems. The behaviour of the contact forces between a plate and the sprocket in the Analogue Huygens Pendulum should be similar to that of an automotive system; therefore the model can be used further for applications in this direction. Overall, one can conclude that the contact between the plates and the sprockets produce elastic-plastic impacts. This understanding represents a step in improving the I.T. chain properties and the I.T. chain systems as a whole.

\section{ACKNOWLEDGMENT}

This work was partially supported by the strategic grant POSDRU/159/1.5/S/137070 (2014) of the Ministry of Labour, Family and Social Protection, Romania, co-financed by the European Social Fund - Investing in People, within the Sectorial Operational Programme Human Resources Development 2007-2013.

\section{REFERENCES}

[1] R. V. Mulik and M. M. Joshi, "Dynamic Analysis of timing chain system of a high speed three cylinder diesel engine," Research Invent International Journal of Engineering and Science, vol. 4, no. 5, May 2014, pp. 21-25.

[2] I. Masao and W. Hiroyuki, "Analysis and control of inverted tooth chain vibration," World Automotive Congress FISITA, Budapest, 30 May-June 2010, pp. 852-861. 
[3] A. Emmerson, "Things are seldom what they seem Christiaan Huygens, the pendulum and the cycloid," NAWCC Bulletin No. 362 June 2006, pp. 295-312.

[4] H. Shan, J. Z. Su, F. Badiu, J. S. Zhu, and L. Xu, "Modeling and simulation of multiple impacts of falling rigid bodies," Mathematical and Computer Modelling, no. 43, 2006, pp. 592-611.

[5] A. A. Shabana, Computational Dynamics, $2^{\text {nd }}$ edition, John Wiley \& Sons, New York, 2000.

[6] A. A. Shabana, Dynamics of Multibody Systems, $4^{\text {th }}$ edition, University of Illinois, Chicago, Cambridge University Press, 2013.

[7] F. Mirouche, "Fundamentals of multibody dynamics theory and applications," Birkhauser Basel, 2006.

[8] MSC Software. MSC Adams 2008 Release Guide. [Online]. Available: www.mscsoftware.com

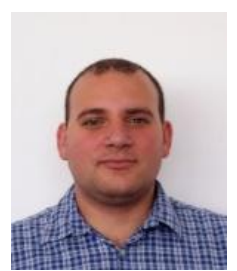

Karim Shalaby was born in Cairo, Egypt, in 1984. He obtained his bachelor degree in mechatronics from the Faculty of Mechanics, H.T. I. of 10th of Ramadan, Egypt in 2008, and his master degree in mechatronics from Transylvania University of Brasov, Romania, in 2011. At present, Mr. Shalaby is a Ph.D. student in mechnical engineering at Transylvania University of Brasov. He was trained in Bahgat Group, Egypt, in P.C.B manufacturing (01.05.2006 - 01.08.2006). After he graduated from the university, he worked as an electro-mechanical engineer in Optima RPM, Egypt, (10.10.2008 - 15.06.2009), being responsible for the electro-mechanical systems in construction fields. Currently he works as an engineer at Schaeffler Romania. He is interested in analyzing the vibration of the I. T. chain systems in order to reduce the vibrations.

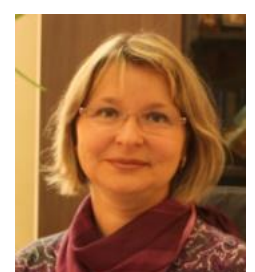

Simona Lache was born in 1968. She holds a degree in mechanical engineering (precision mechanics) at UTBv, an European master in energy management and mechanical engineering and obtained her Ph.D. in 2000 , in the field of structural dynamics. She has been an academic staff at UTBv since graduation, a full professor since 2006 and the $\mathrm{PhD}$ supervisor since 2007.

Prof. Lache is an author/co-author for 7 books, 50 articles published in journals indexed in international databases, 22 scientific papers presented in international conferences and published in ISI Proceedings. She is a co-author for a patent and for other 2 proposals submitted for evaluation at the national organization for patents. Currently S. Lache coordinates $2 \mathrm{Ph} . \mathrm{D}$. students and is mentor of one post-doctoral researcher. Since 2008, other two doctoral students obtained their Ph.D. degree under her supervision. She holds the position of the vice-rector since 2008, being responsible for internationalization and quality assurance.

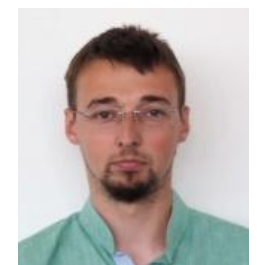

Florin V. Corciova was born in Brasov, Romania, in 1989. He obtained his bachelor degree in physical engineering from Transylvania University of Brasov, Faculty of Engineering and Industrial Management in 2012 and a master degree in innovative fabrication, also from Transylvania University of Brasov, in 2014. He was trained in benchmark electronics SRL in electrical and electronic manufacturing industry (12.2011- 04.2012). Currently, he works at Schaeffler Romania, being responsible for technical analyses. He is interested in test devices development. 